\title{
Terahertz Detection of Zika Viruses
}

\author{
Subhashish Dolai and Massood Tabib-Azar \\ ECE Department, University of Utah \\ Salt Lake City, UT 841012
}

\begin{abstract}
Our main objective in this work was to examine the possibility of non-intrusive, label-free, detection of whole Zika viruses using terahertz signals with or without a targeting/binding oligonucleotide (aptamers). We report for the first time the use of terahertz electromagnetic waves $(0.75 \mathrm{THz}-1.1 \mathrm{THz})$ to detect Zika viruses. The Zika/aptamer complexes showed a reproducible terahertz reflection coefficient minimum at $1.064 \mathrm{THz}$ while the Zika virus's reflection minimum was at $1.073 \mathrm{THz}$. Of different substrates we examined, the polyester petri dish provided a very low loss and excellent terahertz transmission. To increase the interaction between the terahertz signal and the sample we also used polyester microbeads coated with aptamers. We then measured the terahertz reflection from the microbeads as a function of Zika concentration. The resulting terahertz Zika sensor had sensitivity of 63 $\mathrm{Hz} / \mathrm{Zika}$ and minimum detectable signal of $\sim 16 \times 10^{3}$ Zika. Other substrates such as Graphene on polyethylene terephthalate (PET), $50 \mathrm{~nm}$-thick gold film on polycarbonate, thin ( $30 \mu \mathrm{m}$-thick) glass slide and Teflon were also examined. Graphene substrate enabled direct detection of the Zika without any aptamers.
\end{abstract}

Keywords: electromagnetic properties of viruses, terahertz sensors, label-free detection of viruses, nanotechnologies

Introduction: Terahertz signals with $\sim 300 \mu \mathrm{m}$ wavelengths provide a good balance between penetration depth and spatial resolution in biological applications[1][2]. Plasmonic terahertz structures [3] and microfluidics with terahertz waveguides can be readily fabricated using $3 \mu \mathrm{m}$ photolithography and routine fabrication steps. With photon energies in the $\sim \mathrm{meV}$ range $(4.14 \mathrm{meV}$ at $1 \mathrm{THz})$, terahertz waves can excite some of the internal degrees of freedom in biological and related molecules such as $\mathrm{H}_{2} \mathrm{O}$ and $\mathrm{CO}_{2}$ [4][5]. Glucose, yeast [6][7], proteins[8]-[10], oligonucleotides [11][12] , and even viruses[13] are studied using terahertz spectroscopy. In most sensing applications, structures to focus the terahertz electromagnetic signal in the form of bowtie antenna or plasmonic structures are used. Viruses are usually detected using their DNA with polymerase chain reaction (PCR)[14]-[19]. Other techniques involving antigens and aptamers have also been developed in the past and along with fluorescent readout mechanisms provide a faster route for sensing. For the first time we use terahertz reflection spectroscopies, without plasmonics to detect Zika virus and it's binding with an aptamer designed to target its capsid proteins. Aptamers are oligonucleotides designed to target and bind to a specific molecule, tissue, biomarker or a pathogen.

Our main goal here is to either directly detect the Zika virus through its signature terahertz absorption lines or to bind aptamers with required number of linkers and a suitable substrate to enable direct and selective detection of the Zika-aptamer complexes. Aptamers are oligonucleotides design to target and bind to specific molecules[20], [21]. Aptamers possess high target affinity, better selectivity and stability than antibodies, more cost effective, and they can be recycled for continuous sensor use. Here we use an aptamer that was designed to bind to the capsid proteins of Zika virus to form aptamer-Zika complexes and to capture the Zika virus on (thin layer metallic) substrates for more efficient interrogation using terahertz signals. The sensors discussed here can be easily adopted to detect other viruses such as coronavirus by using their corresponding aptamers or antibodies.

Material and Methods: Terahertz spectroscopy used in this work is performed using an automated network analyzer shown in Figs. 1a and 1b. The biofluid sample was deposited directly on a polyster substrate. Fig. 2 schematically shows different sample holder and reflection measurement modes we used to sense the Zika virus. To selectively bind the Zika virus to the substrate, we used an aptamer with thiol end group [22] (molecular weight of $10 \mathrm{kDa}\left(1.66 \times 10^{-20} \mathrm{gm}\right)$ form BasePair Biotechnologies Inc), that binds with Zika SF9 envelope protein [23]. The aptamer was activated as per instructions from the supplier. 
The steps involved using resuspension buffer (BasePair product) for emulsification of the dried aptamer, dilution to a working concentration of $100 \mu \mathrm{M}$ using the aptamer folding buffer, and heating to $90-95{ }^{\circ} \mathrm{C}$ for 5 minutes. The folded aptamer was then further diluted to $1 \mu \mathrm{M}$ solution using 1x Phosphate buffer saline (PBS) and $1 \mathrm{mM}$ Magnesium Chloride $\left(\mathrm{MgCl}_{2}\right)$ solutions.
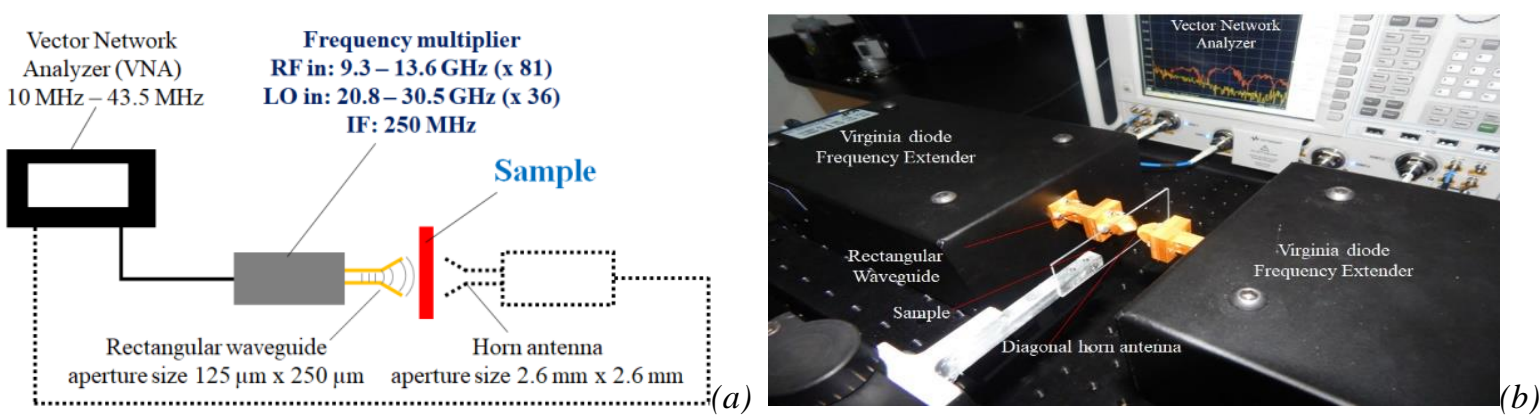

Fig 1: a) Experimental setup used in the terahertz measurements to detect and identify the Zika virus. b) Image of the experimental setup.

The heat inactivated Zika virus was obtained from Zeptomatrix Inc with the stock solution having a concentration of TCID50/ml titer [24]. The estimated number of Zika in $2 \mu 1$ stock solution was $\sim 3.5 \times 10^{8}$ [25]. Zika virus is a sphere with $40 \mathrm{~nm}$ diameter (mass: $43 \mathrm{kDa}$ or $7.1 \times 10^{-20} \mathrm{gm}$ ). The $\mathrm{pH}$-neutral buffer solutions containing the aptamer and the Zika virus were deposited on the dielectric substrates with a spacer of $70 \mu \mathrm{m}$ (Fig. 2a), with graphene substrate in (Fig 2b) and a polycarbonate substrate having well structure coated with $50 \mathrm{~nm}$ layer of gold. In the last case, the Zika was added to the aptamer/gold substrate along-with aptamer coated $0.5 \mu \mathrm{m}$ microbeads (obtained from Polysciences). The thin gold layer was used to ensure proper attachment of the thiolated aptamer and Zika.

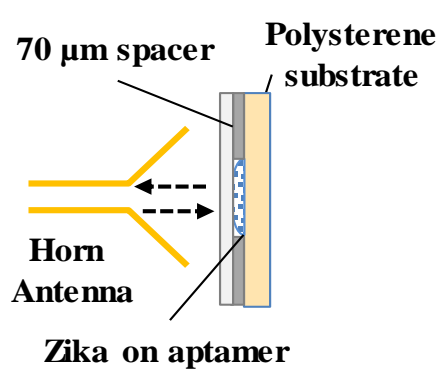

(a)

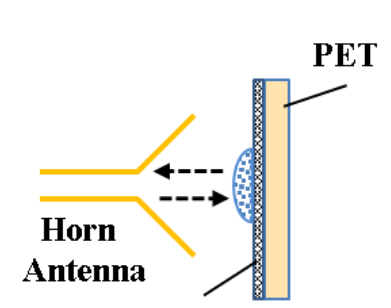

Graphene (b)

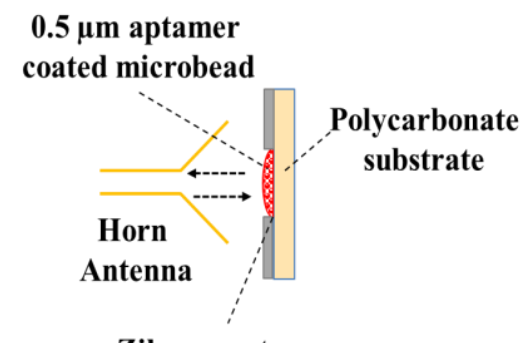

Zika on aptamer

(c)

Fig 2: Schematics of the reflection mode measurements used in this work. a) A spacer was used to ensure constant Zika/aptamer thickness. b) A graphene layer was used to provide a reflection reference and amplify the effect of analyte residual charge through changing its energy band gap. (c) Polycarbonate substrate with $0.5 \mu \mathrm{m}$ microbeads on Zika to measure terahertz properties for different concentration of Zika.

Results and Discussions: We studied the reflection coefficient $\left(\mathrm{S}_{11}\right)$ spectra using the Terahertz VNA setup shown in Fig. 1. Fig. 2 shows the measurement arrangements we used. The $S_{11}$ spectra of bare substrate, aptamer $(5 \mu \mathrm{l})$, Zika $(5 \mu \mathrm{l})$ and Zika/aptamer $(5 \mu \mathrm{l}$ and $5 \mu \mathrm{l})$ fluids placed in the gap region with spacer (Fig. 2a) is shown in Fig. 3a. The individual dip in the reflection curve (averaged 30 points) can be seen for each of the different materials with bare substrate at $1.036 \mathrm{THz}$, in aptamer-coated substrate at $1.066 \mathrm{THz}$, Zika-coated substrate at $1.074 \mathrm{THz}$, and in the Zika/aptamer-coated substrate at $1.065 \mathrm{THz}$. The shift in the reflection spectra are shown in Fig. $3 \mathrm{~b}$ and were large enough to resolve the difference between aptamer-, Zika/aptamer- and Zika-coated substrates. 


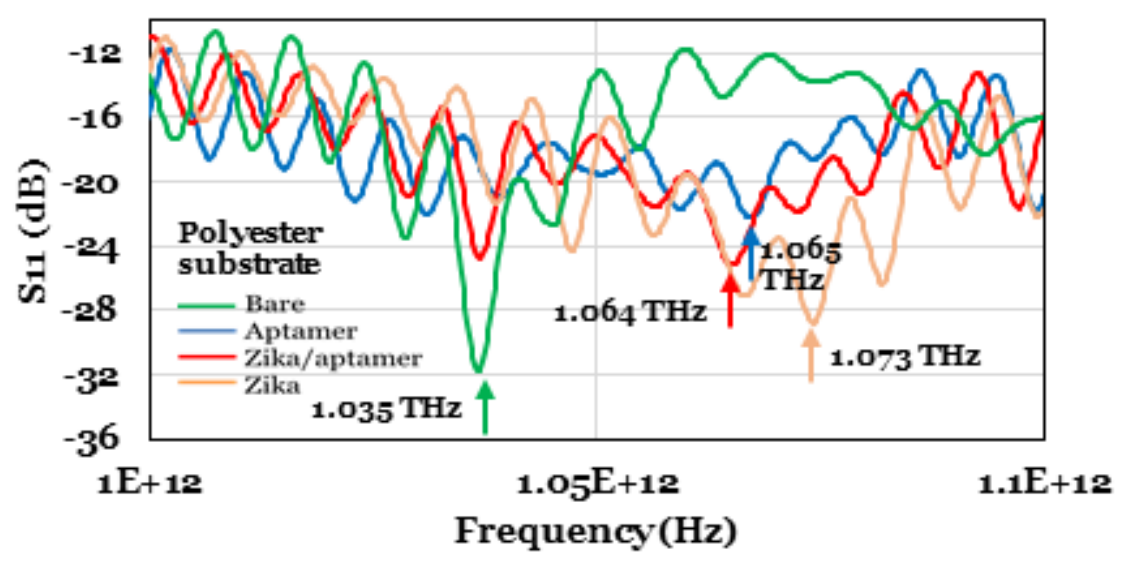

(a)

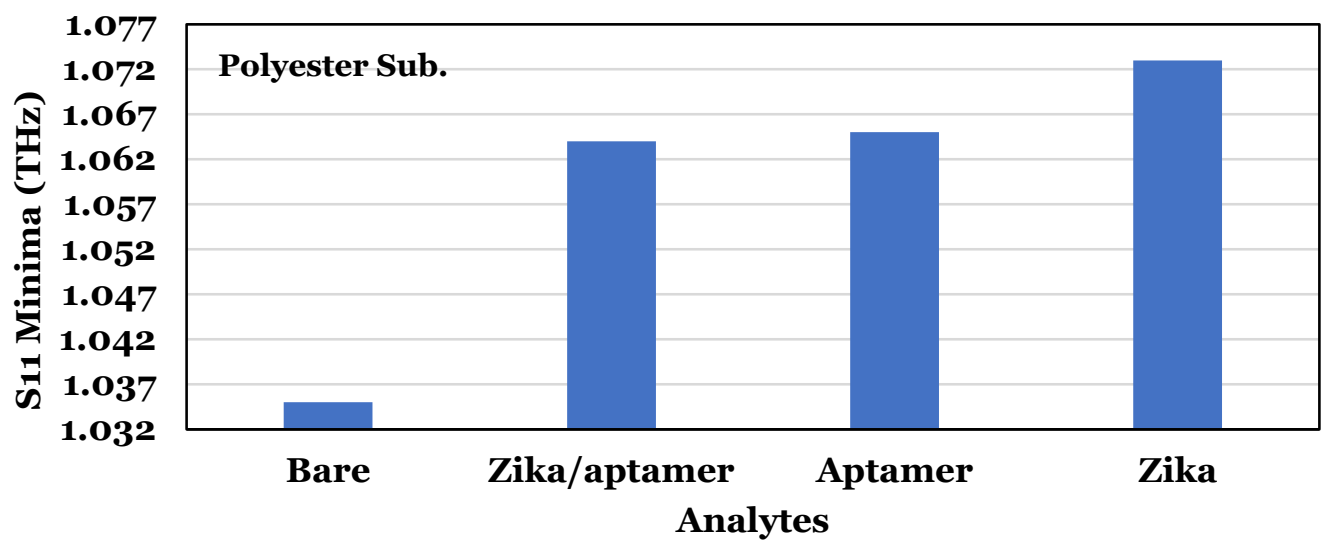

(b)

Fig 3: a) Terahertz reflection $\left(S_{11}\right)$ properties of aptamer, aptamer/Zika and Zika on polyester substrate. b) Reflection spectra minima of different materials on polyester substrate.

Similar set of measurements were also carried out on a monolayer of graphene on polyethylene terephthalate (PET). Graphene has zero band gap energy with $0.34 \mathrm{~nm}$ thickness, and very high electron mobility of $\sim 10,000 \mathrm{~cm}^{2} \mathrm{~V} / \mathrm{s}$ [26]-[29]. Its band-gap energy can be change using an electric field or charges nearby. The most important reason for using graphene was to examine the effect of the negative residual charges that aptamers and Zika carry on the reflection coefficient of the graphene layer. The idea is that if the graphene's conductivity changes in the presence of the Zika virus, we may be able to use it as a sensing material for Zika detection without a need for aptamers. Fig. 4a shows that the terahertz coefficient spectrum has a clear minimum that shifts depending on the aptamer, Zika, and aptamer/Zika conductivity and dielectric constant. The Zika/aptamer complex appears to be acting as a dielectric loading on the graphene shifting the reflectance minimum to lower frequencies (from 1.072 for graphene to 1.071 $\mathrm{GHz}$ for Zika/aptamer on graphene). Zika shifted the minimum to $1.085 \mathrm{GHz}$ while aptamer shifted it to $1.086 \mathrm{GHz}$. Using a simple reflection coefficient equation of $S_{11}=\frac{Z_{L}-Z_{0}}{Z_{L}+Z_{0}}$, and $f_{\min }=\frac{1}{2 \pi} \sqrt{\frac{1}{\varepsilon \mu}}$ where $\mu$ is the permeability $\left(=\mu_{0}\right)$ and $\varepsilon$ is the permittivity. We also note that $Z_{L}=\sqrt{\frac{\mu}{\varepsilon}}$. Thus from the changes in the $\mathrm{S}_{11}$ and $f_{\min }$ we determined the Zika/aptamer relative dielectric constant to be 2.9 (that of graphene at $1 \mathrm{THz}$ is 2.56) and the terahertz graphene resistance change due to Zika was $26 \Omega$ and its change due to the aptamer was $12 \Omega$. The increase in the graphene resistance can be attributed to the change in its bandgap energy of $\Delta \mathrm{E}_{\mathrm{g}} \sim 0.8 \mathrm{meV}$ when Zika was deposited and $\Delta \mathrm{E}_{\mathrm{g}} \sim 1.6 \mathrm{meV}$ when aptamer was deposited. 


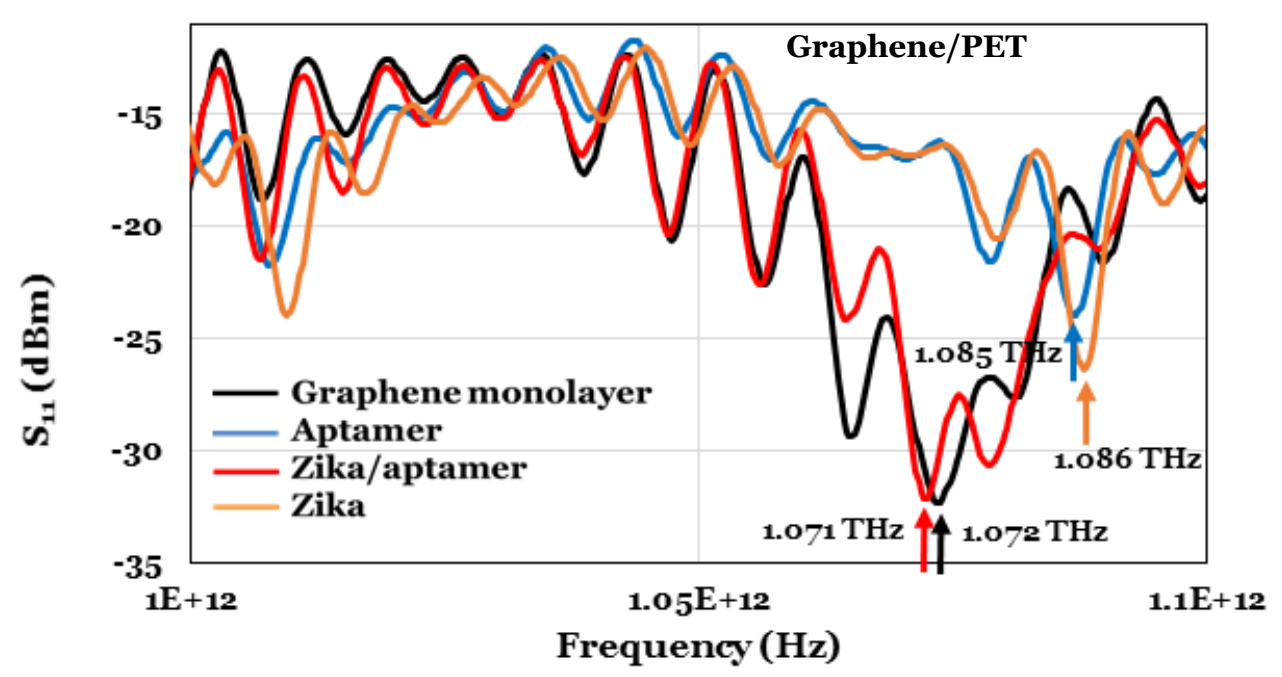

(a)

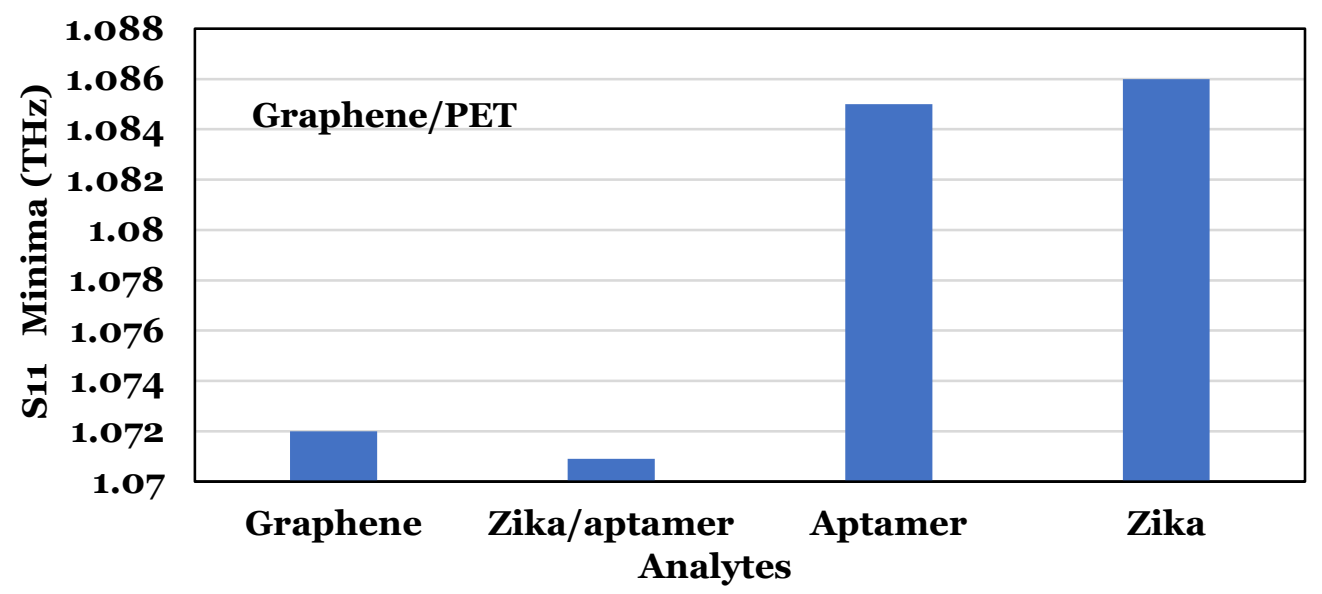

Fig 4: a) Terahertz reflection coefficient of different aptamer and Zika on graphene. b) Terahertz reflection minima for different analytes on graphene.

The sensitivity measurement of Zika binding with aptamer was done with respect to the coverage of $0.5 \mu \mathrm{m}$ aptamer coated microbeads with Zika on aptamer coated gold substrate (polycarbonate). The polycarbonate well (Fig 5(a)) was first sputtered with thin layer of gold $\sim 50 \mathrm{~nm}$ and then subsequently aptamer coated by applying $2 \mu \mathrm{l}$ aptamer $(1 \mu \mathrm{M})$ and placed at $60^{\circ} \mathrm{C}$ for 2 minutes. The excess aptamer was cleaned with DI water bath. The aptamer coated beads were placed in the same well with pre-coated aptamer along-with Zika virus added in increasing concentration and then heated at $50{ }^{\circ} \mathrm{C}$ for 5 minutes. The Zika virus acts as a bridge (schematically shown in Fig 5(b)) between the sputtered gold/aptamer on the substrate and the aptamer/microbeads. The excess microbeads were cleaned by placing it in water bath. The terahertz reflection spectra for increasing concentration of stock Zika virus have been shown in Fig 5(c). With the increasing concentration of Zika virus, the coverage of the microbeads increased on the substrate and the reflection spectrum minima shifted towards the left. The reflection coefficient minima have been shown in Fig 5(d) starting with bare gold substrate and increasing Zika concentration. The sensitivity is defined as, $S=\frac{\Delta f}{\Delta C_{Z i k a}}$, where $\mathrm{f}$ is the frequency minima of the reflection spectra and $C_{Z i k a}$ is the Zika concentration. The frequency minima shifted negligibly till Zika concentration reached a threshold at $3 \mu \mathrm{l}$. The largest sensitivity was observed for Zika concentration from $4 \mu \mathrm{l}$ to $5 \mu \mathrm{l}$. After 5 $\mu \mathrm{l}$, the response saturated. The highest sensitivity $63 \mathrm{~Hz} / \mathrm{Zika}$ with the minimum detectable signal of $\sim 16 \times 10^{3}$ Zika were obtained from these measurements. 


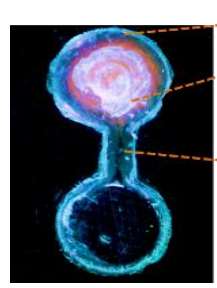

Well structure

Aptamer/microbead on Zika

Polycarbonate substrate

(a)

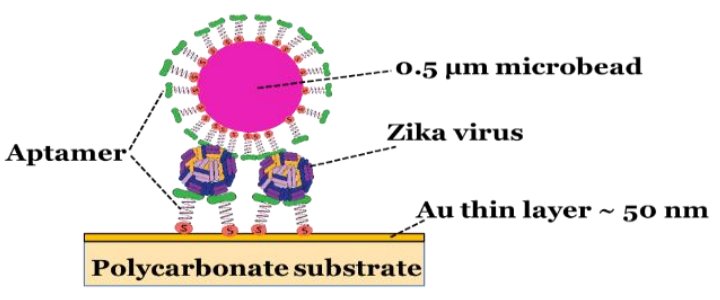

(b)

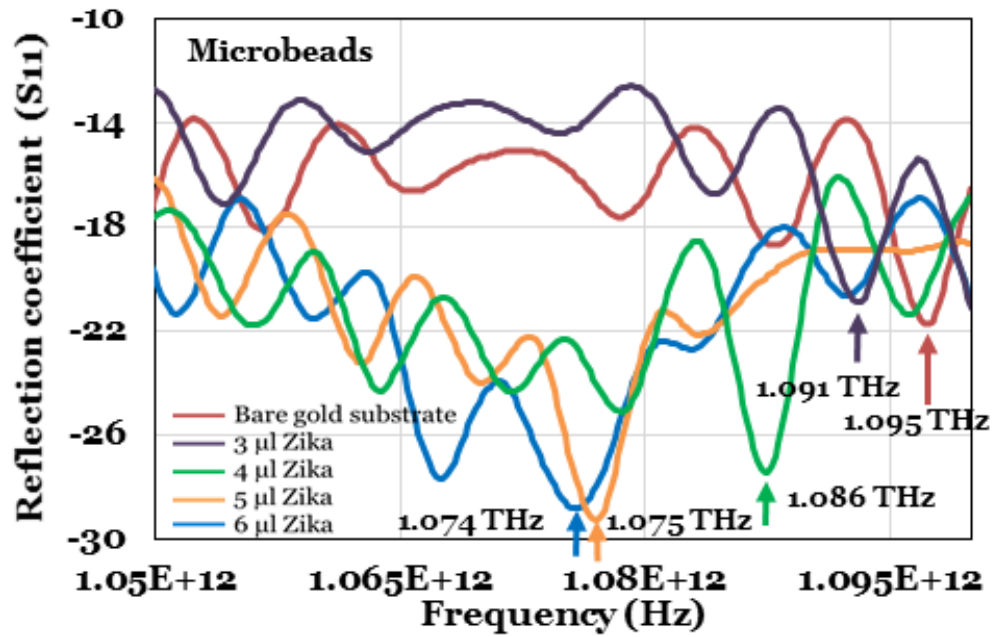

(c)

Fig5: a) Optical photograph of the aptamer coated microbeads on Zika on polycarbonate substrate having well structure. The well structure having the microbeads attached have thin layer of gold ( 50 $n m)$ and then aptamer deposited. b) Schematic of the Zika virus as a bridge between the aptamer coated microbead and aptamer covered gold coated polycarbonate substrate. c) Terahertz forward reflection coefficient of aptamer coated microbeads at different concentration of Zika.

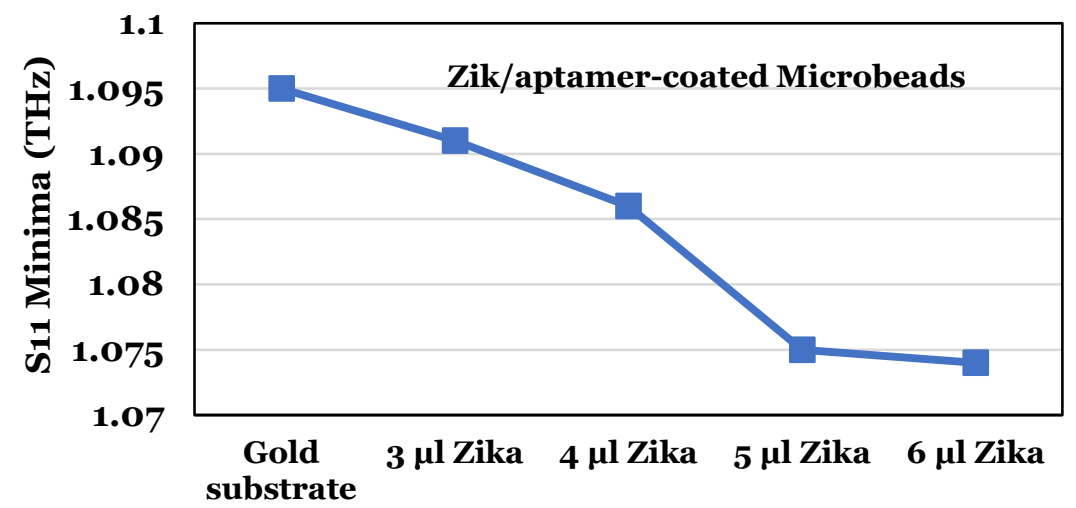

Zika Added to Microbeads

(a)

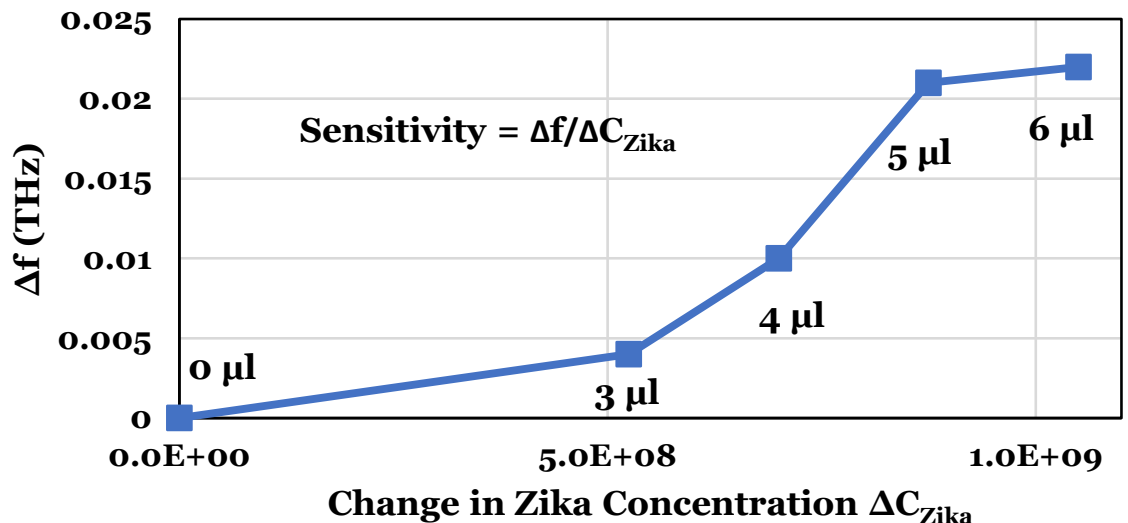


Fig. 6: a) Reflection coefficient minima frequency as a function of Zika concentration in aptamer-coated microbead ample. b) Sensitivity of the terahertz measurement as a function of Zika concentration in the microbead sample.

Other substrates were also examined. Glass had a large absorption at terahertz frequencies while Teflon had very low absorption. Teflon could not be conveniently used because analytes did not stick to it. The microbeads were deposited on a thin layer of gold on polycarbonate substrate. The thin layer of gold enabled attachments of thiolated aptamer.

Conclusion: Our main objective in this work is to examine the possibility of non-intrusive, label-free, detection of whole Zika viruses using terahertz signals with or without a targeting/binding oligonucleotide (aptamers). We report for the first time the use of terahertz electromagnetic waves $(0.75 \mathrm{THz}-1.1 \mathrm{THz})$ to detect Zika viruses. The Zika/aptamer complexes showed a reproducible terahertz reflection coefficient minimum at $1.064 \mathrm{THz}$ while the Zika virus's reflection minimum was at $1.073 \mathrm{THz}$. Of different substrates we examined, the polyester petri dish provided a very low loss and excellent terahertz transmission. To increase the interaction between the terahertz signal and the sample we also used polyester microbeads coated with aptamers. We then measured the terahertz reflection from the microbeads as a function of Zika concentration. The resulting terahertz Zika sensor had sensitivity of 63 $\mathrm{Hz} /$ Zika and minimum detectable signal of $\sim 16 \times 10^{3}$ Zika. Other substrates such as Graphene on polyethylene terephthalate (PET), $50 \mathrm{~nm}$-thick gold film on polycarbonate, thin (30 mm-thick) glass slide and Teflon were also examined. Graphene substrate enabled direct detection of the Zika without any aptamers.

\section{Acknowledgement:}

This research was supported by a National Science Foundation EAGER Grant (\#1931100) under Dr. Leon Esterowitz. The authors also acknowledge contributions of Dr. Jules Magda and Dr. Tram Nguyen, Chemical Engineering Department, University of Utah, for Aptamer activation and Zika virus handling and processing.

\section{Reference:}

[1] D. Cheng et al., "Terahertz biosensing metamaterial absorber for virus detection based on spoof surface plasmon polaritons," Int. J. RF Microw. Comput. Eng., vol. 28, no. 7, Sep. 2018.

[2] X. Yang, K. Yang, Y. Luo, and W. Fu, "Terahertz spectroscopy for bacterial detection: opportunities and challenges," Applied Microbiology and Biotechnology, vol. 100, no. 12. Springer Verlag, pp. 5289-5299, 01-Jun-2016.

[3] M. Borovkova, M. Khodzitsky, O. Cherkasova, A. Popov, and I. Meglinski, "Towards noninvasive reflection measurement of water content in biotissue by means of terahertz timedomain spectroscopy," Proc. - Int. Conf. Laser Opt. 2018, ICLO 2018, p. 524, 2018.

[4] M. Grechko et al., "Coupling between intra-and intermolecular motions in liquid water revealed by two-dimensional terahertz-infrared-visible spectroscopy," Nat. Commun., vol. 9, no. 1, 2018.

[5] H. Hoshina, Y. Iwasaki, E. Katahira, M. Okamoto, and C. Otani, "Structure and dynamics of bound water in poly(ethylene-vinylalcohol) copolymers studied by terahertz spectroscopy," Polymer (Guildf)., vol. 148, pp. 49-60, Jul. 2018.

[6] O. Fawole, K. Sinha, and M. Tabib-Azar, "Monitoring yeast activation with sugar and zero-calorie sweetener using terahertz waves," in 2015 IEEE SENSORS - Proceedings, 2015.

[7] O. Fawole and M. Tabib-Azar, "Terahertz quantification of ethanol and sugar concentrations in water and its application for noninvasive real-time monitoring of fermentation," in IEEE MTT-S International Microwave Symposium Digest, 2016, vol. 2016-Augus.

[8] S. J. Kim, B. Born, M. Havenith, and M. Gruebele, "Real-time detection of protein-water dynamics upon protein folding by terahertz absorption spectroscopy," Angew. Chemie - Int. Ed., vol. 47, no. 34, pp. 6486-6489, Aug. 2008.

[9] H. Yoshida et al., "Terahertz sensing method for protein detection using a thin metallic mesh," Appl. Phys. Lett., vol. 91, no. 25, 2007. 
[10] S. Ebbinghaus et al., "Protein sequence- and pH-dependent hydration probed by terahertz spectroscopy," J. Am. Chem. Soc., vol. 130, no. 8, pp. 2374-2375, Feb. 2008.

[11] M. Tang et al., "Terahertz spectroscopy of oligonucleotides in aqueous solutions," J. Biomed. Opt., vol. 20, no. 9, p. 095009, Sep. 2015.

[12] N. T. Bagraev, A. L. Chernev, L. E. Klyachkin, A. M. Malyarenko, A. K. Emel'yanov, and M. V. Dubina, "Terahertz response of DNA oligonucleotides on the surface of silicon nanostructures," Semiconductors, vol. 50, no. 9, pp. 1208-1215, Sep. 2016.

[13] S. J. Park, S. H. Cha, G. A. Shin, and Y. H. Ahn, "Sensing viruses using terahertz nano-gap metamaterials," Biomed. Opt. Express, vol. 8, no. 8, p. 3551, Aug. 2017.

[14] K. O. Murray et al., "Prolonged detection of zika virus in vaginal secretions and whole blood," Emerg. Infect. Dis., vol. 23, no. 1, pp. 99-101, Jan. 2017.

[15] G. Calvet et al., "Detection and sequencing of Zika virus from amniotic fluid of fetuses with microcephaly in Brazil: a case study," Lancet Infect. Dis., vol. 16, no. 6, pp. 653-660, Jun. 2016.

[16] O. Faye, O. Faye, A. Dupressoir, M. Weidmann, M. Ndiaye, and A. Alpha Sall, "One-step RTPCR for detection of Zika virus," J. Clin. Virol., vol. 43, no. 1, pp. 96-101, Sep. 2008.

[17] A. C. Gourinat, O. O'Connor, E. Calvez, C. Goarant, and M. Dupont-Rouzeyrol, "Detection of zika virus in urine," Emerg. Infect. Dis., vol. 21, no. 1, pp. 84-86, 2015.

[18] C. Klungthong et al., "Dengue virus detection using whole blood for reverse transcriptase PCR and virus isolation," J. Clin. Microbiol., vol. 45, no. 8, pp. 2480-2485, Aug. 2007.

[19] D. Musso, C. Roche, T. X. Nhan, E. Robin, A. Teissier, and V. M. Cao-Lormeau, "Detection of Zika virus in saliva," J. Clin. Virol., vol. 68, pp. 53-55, Jul. 2015.

[20] R. D. Jenison, S. C. Gill, A. Pardi, and B. Polisky, "High-resolution molecular discrimination by RNA,” Science (80-. )., vol. 263, no. 5152, pp. 1425-1429, 1994.

[21] K. H. Lee and H. Zeng, "Aptamer-Based ELISA Assay for Highly Specific and Sensitive Detection of Zika NS1 Protein," Anal. Chem., vol. 89, no. 23, pp. 12743-12748, Dec. 2017.

[22] "Base Pair Biotechnologies - Aptamer Discovery Company." [Online]. Available: https://www.basepairbio.com/. [Accessed: 04-Sep-2019].

[23] "Zika Envelope Recombinant Antigen | Zika Virus | ProSpec." [Online]. Available: https://www.prospecbio.com/zika_envelope_sf9. [Accessed: 04-Sep-2019].

[24] R. N. Charrel, I. Leparc-Goffart, S. Pas, X. de lamballerie, M. Koopmans, and C. Reusken, "Background review for diagnostic test development for Zika virus infection," Bull. World Health Organ., vol. 94, no. 8, pp. 574-584D, 2016.

[25] S. Dolai and M. Tabib-Azar, " $433 \mathrm{MHz}$ Lithium Niobate microbalance aptamer-coated whole Zika virus sensor with $370 \mathrm{~Hz} / \mathrm{ng}$ sensitivity," IEEE Sens. J., pp. 1-1, 2019.

[26] Shao, Y., Wang, J., Wu, H., Liu, J., Aksay, I. A., \& Lin, Y. (2010). Graphene based electrochemical sensors and biosensors: a review. Electroanalysis: An International Journal Devoted to Fundamental and Practical Aspects of Electroanalysis, 22(10), 1027-1036.

[27] Huang, Y., Dong, X., Shi, Y., Li, C. M., Li, L. J., \& Chen, P. (2010). Nanoelectronic biosensors based on CVD grown graphene. Nanoscale, 2(8), 1485-1488.

[28] Kuila, T., Bose, S., Khanra, P., Mishra, A. K., Kim, N. H., \& Lee, J. H. (2011). Recent advances in graphene-based biosensors. Biosensors and bioelectronics, 26(12), 4637-4648.

[29] Xu, W., Xie, L., Zhu, J., Tang, L., Singh, R., Wang, C., \& Ying, Y. (2019). Terahertz biosensing with a graphene-metamaterial heterostructure platform. Carbon, 141, 247-252. 